\title{
Apigenin reduces the excessive accumulation of lipids induced by palmitic acid via the AMPK signaling pathway in HepG2 cells
}

\author{
JING LU ${ }^{1}$, ZHUOQUN MENG ${ }^{1}$, BIJUN CHENG ${ }^{1}$, MEITONG LIU $^{1}$, SIYU TAO $^{2}$ and SHUANG GUAN ${ }^{1}$ \\ ${ }^{1}$ Department of Food Quality and Safety, College of Food Science and Engineering, Jilin University, Changchun, Jilin 130062; \\ ${ }^{2}$ Department of Pharmacology, College of Basic Medical Sciences, Jilin University, Changchun, Jilin 130021, P.R. China
}

Received November 7, 2018; Accepted May 23, 2019

DOI: $10.3892 /$ etm.2019.7905

\begin{abstract}
In recent years, increasing attention has been paid to diseases caused by excessive accumulation of lipids in the liver with therapeutic agents derived from natural products offering an alternative treatment to conventional therapies. Among these therapeutic agents, apigenin, a natural flavonoid, has been proven to exert various beneficial biological effects. In the present study, the antiadipogenic effects of apigenin in HepG2 cells was investigated. It was demonstrated that the treatment of cells with different concentrations of apigenin for $24 \mathrm{~h}$ significantly decreased the palmitic acid-induced increases in total cholesterol (TC) and triglyceride (TG) levels as well as intracellular lipid accumulation. In addition, apigenin increased the phosphorylated-AMP-activated protein kinase (AMPK) levels but decreased the expression levels of 3-hydroxy-3-methylglutaryl CoA reductase, sterol regulatory element-binding protein (SREBP)-1, fatty acid synthase, and SREBP-2 in a concentration-dependent manner. The present findings suggested that apigenin might improve lipid metabolism by activating the AMPK/SREBP pathway to reduce lipid accumulation in the liver.
\end{abstract}

\section{Introduction}

In recent years, obesity has received increasing attention because it leads to various metabolic diseases, such as hyperlipidemia, hypertension, atherosclerosis and type II diabetes mellitus (1). The liver is an important organ associated with lipid metabolism. Notably, increases in lipid metabolism is mainly observed in the liver, which can lead to chronic diseases related to hepatocytes (2). If left untreated, this increase in metabolism can lead to hepatocellular carcinoma therefore, it is necessary to investigate the underlying

Correspondence to: Professor Shuang Guan, Department of Food Quality and Safety, College of Food Science and Engineering, Jilin University, 5333 Xi'an Road, Changchun, Jilin 130062, P.R. China E-mail: gshuang1973@126.com

Key words: apigenin, palmitic acid, lipid accumulation, AMP-activated protein kinase, compound C molecular mechanism (3). Adipogenesis is regulated via a complex process involving coordinated changes in hormone sensitivity and gene expression regulated by various transcription factors (4). The sterol regulatory element-binding protein (SREBP) family of transcription factors have pivotal roles in the regulation of cellular lipid metabolism (5). SREBP-1 mainly regulates the metabolism of fatty acids and triglycerides, whilst SREBP-2 regulates the biosynthesis of cholesterol (6). Fatty acid synthase (FAS), a protein related to fat synthesis, is regulated by SREBP-1 (7). SREBP-2 regulates 3-hydroxy-3-methylglutaryl CoA reductase (HMGCR) expression and thereby catalyzes the rate-limiting step of cholesterol biosynthesis (8). Furthermore, AMP-activated protein kinase (AMPK) is an important protein involved in energy metabolism and has a significant role in the regulation of fatty acid metabolism. An in vitro study determined that alliin attenuates adipogenesis induced by 1,3-dichloropropan-2ol in HepG 2 cells by activating the AMPK/SREBP signaling pathway (9). Therefore, it is reasonable to speculate that the AMPK/SREBP pathway might be important for the amelioration of lipogenesis during palmitic acid treatment.

For the development of novel antilipidemic drugs with high efficacy and few adverse effects, attention has gradually shifted to the natural plant flavonoids in recent years. Apigenin, a type of plant flavonoid with the chemical name 4',5,7-dihydroxyflavone, is recognized as a bioactive flavonoid that has antioxidant, anticancer and anti-inflammatory properties that can also lower blood pressure (10-12). In particular, a previous study has demonstrated that apigenin can alleviate myocardial hypertrophy and the hypertrophy-induced glucose and lipid metabolism abnormalities in rats (13). In addition, apigenin alleviates glucose metabolic disorder induced by a high-fat diet in 20-week-old mice (14). It still remains unclear whether apigenin has a hypolipidemic effect, or whether the AMPK/SREBP pathway is the major determinant of anti-adipogenic activity. Palmitic acid is a saturated high-grade fatty acid that is widely distributed in animal and vegetable oils in the form of glycerides, and is commonly used to establish lipotoxic in vitro and in vivo models (15).

The present study established a common hyperlipidemia model by using appropriate doses of palmitic acid to determine whether apigenin lowered lipid levels and, to explore the underlying mechanism in depth. 


\section{Materials and methods}

Reagents. Palmitic acid and dimethyl sulfoxide (DMSO) were purchased from Sigma-Aldrich; Merck KGaA. The apigenin standard was purchased from Beijing Solarbio Technology Co. The AMPK inhibitor compound $\mathrm{C}$ was purchased from Selleck Chemicals LLC. Anti-phospho-AMPK (P-AMPK; Thr172; cat. no. 2535) was purchased from Cell Signaling Technology, Inc., anti-AMPKa1 (cat. no. 10929-2-AP), SREBP-2 (cat. no. 14508-1-AP), FAS (cat. no. 13098-1-AP), HMGCR (cat. no. 13533-1-AP) and $\beta$-actin (cat. no. 66009-1-lg) were purchased from Proteintech Group Inc., SREBP-1 (cat. no. ab3259) was purchased from Abcam. Horseradish peroxidase (HRP)-conjugated goat anti-mouse $\operatorname{IgG}(\mathrm{H}+\mathrm{L}$; cat. no. SA00001-1) and HRP-conjugated goat anti-rabbit $\operatorname{IgG}(\mathrm{H}+\mathrm{L}$; cat. no. SA00001-2) secondary antibodies were obtained from Proteintech Group Inc.

Cell culture and treatment. Liver cancer HepG2 cells, purchased from the American Type Culture Collection, were cultured in high-glucose DMEM (GE Healthcare Life Sciences) with $10 \%$ fetal bovine serum (Clark Bioscience) and $1 \%$ penicillin/streptomycin at $37^{\circ} \mathrm{C}$ in an atmosphere containing $5 \% \mathrm{CO}_{2}$. The cells were dissociated with $0.25 \%$ trypsin (w/v) and $0.52 \mathrm{mM}$ EDTA [M\&C Gene Technology (Bejing) Ltd.] and routinely sub-cultured at $80 \%$ confluency.

Cell cultures were treated with various concentrations of apigenin in DMSO as the carrier solvent. Palmitic acid binds to fatty-acid-free BSA (Beijing Solarbio Science \& Technology Co., Ltd.). In brief, palmitic acid was dissolved in $1 \mathrm{X}$ PBS and a $250 \mathrm{mM}$ stock solution was obtained following various cycles of incubation in a water bath at $70^{\circ} \mathrm{C}$ and vortexing. The stock solution was then added to serum-free DMEM containing 5\% fatty-acid-free BSA to obtain a $250 \mu \mathrm{M}$ palmitic acid solution, and the resulting diluted solution was used for the cell treatments $(16,17)$. AMPK inhibitor compound $\mathrm{C}$ was diluted with DMSO to a final concentration of $10 \mu \mathrm{M}$ and was used to verify the signaling pathway.

Cell viability assays. Cells were seeded in 96-well plates at a concentration of $1 \times 10^{4}$ cells/well (Corning Inc.) and allowed to adhere overnight at $37^{\circ} \mathrm{C}$. Cells were then treated with different concentrations of apigenin $(0,20,40,80,160,320$, 640 and $1,280 \mu \mathrm{M})$ or palmitic acid $(0,62.5,125,250,500$ and $1,000 \mu \mathrm{M}$ ) for $24 \mathrm{~h}$, and $20 \mu \mathrm{l}$ of MTT (Sigma-Aldrich; Merck $\mathrm{KGaA}$ ) solution $(5 \mathrm{mg} / \mathrm{ml}$ in $1 \% \mathrm{PBS})$ was added to each well and incubated for $4 \mathrm{~h}$ at $37^{\circ} \mathrm{C}$ prior to the end of the culture. The culture medium was subsequently removed and the formazan crystals were dissolved using $150 \mu \mathrm{l}$ DMSO/well. Absorbance values at $570 \mathrm{~nm}$ were determined using a microplate reader (BioTek Instruments, Inc.).

Oil Red $O$ Staining. HepG2 cells were inoculated at a density of $4 \times 10^{5}$ cells per well in 6 -well plates and cultured with different concentrations of apigenin $(10,20$ and $40 \mu \mathrm{M})$ and $250 \mu \mathrm{M}$ palmitic acid for $24 \mathrm{~h}$. Cells were then washed twice with cold $1 \mathrm{X}$ PBS and fixed with $4 \%$ paraformaldehyde for $40 \mathrm{~min}$ at $37^{\circ} \mathrm{C}$. Subsequently, the residual paraformaldehyde was washed with double-distilled water, and cells were incubated with $60 \%$ isopropanol for 3-5 sec. Cells were then stained with freshly prepared $0.5 \%$ Oil Red O solution in $60 \%$ isopropanol for $1 \mathrm{~h}$ at $37^{\circ} \mathrm{C}$ in the dark. Lipid droplets in the cells were observed and imaged using a bright-field microscope (Olympus Corporation) at a magnification of $\mathrm{x} 400$. In addition, the accumulated lipids were extracted with $100 \%$ isopropanol for 3-5 min at room temperature, and the absorbance at $570 \mathrm{~nm}$ was determined using a microplate reader.

Triglyceride (TG) and total cholesterol (TC) assays. HepG2 cells were inoculated at a density of $4 \times 10^{5}$ cells/well in 6-well plates and pretreated with $10 \mu \mathrm{M}$ AMPK inhibitor compound $\mathrm{C}$ for $1 \mathrm{~h}$ at $37^{\circ} \mathrm{C}$ then cultured with different concentrations of apigenin $(10,20$ and $40 \mu \mathrm{M})$ and $250 \mu \mathrm{M}$ palmitic acid for $24 \mathrm{~h}$ at $37^{\circ} \mathrm{C}$ in a culture chamber with $5 \% \mathrm{CO}_{2}$. The intracellular TC and TG contents were measured using a TC assay kit (cat. no. E1015; Applygen Technologies Inc.) and a TG assay kit (cat. no. E1013; Applygen Technologies Inc.) according to the manufacturer's instructions. The absorbance values were measured at $570 \mathrm{~nm}$. Bicinchoninic acid (BCA) protein quantitative kit (Beyotime Institute of Biotechnology) was used to determine the protein concentration of the samples, and the absorbance value of the proteins was measured at $562 \mathrm{~nm}$. The intracellular TC and TG contents are presented as $\mu \mathrm{M} / \mathrm{mg}$ cellular protein.

Western blot analysis. HepG2 cells were seeded in 6-well plates at a concentration of $6 \times 10^{5}$ cells per well and pretreated with $10 \mu \mathrm{M}$ AMPK inhibitor compound $\mathrm{C}$ for $1 \mathrm{~h}$ and then cultured with different concentrations of apigenin (10, 20 and $40 \mu \mathrm{M})$ and $250 \mu \mathrm{M}$ palmitic acid $(16,17)$. The cell pellets were lysed on ice for $30 \mathrm{~min}$ in RIPA buffer (Beyotime Institute of Biotechnology) supplemented with 1:100 PMSF (Beijing Solarbio Science \& Technology Co., Ltd.) according to the manufacturer's specifications and centrifuged at $14,000 \mathrm{x}$ g at $4^{\circ} \mathrm{C}$ for $8 \mathrm{~min}$. The supernatant was then collected and the protein concentration was determined using a $\mathrm{BCA}$ protein concentration assay kit (Beyotime Institute of Biotechnology). An equal amount of protein extract $(80 \mu \mathrm{g})$ was denatured in the sample buffer (Bio-Rad Laboratories, Inc.) at $98^{\circ} \mathrm{C}$ for $10 \mathrm{~min}$ and subsequently separated via 8\% SDS-PAGE and then transferred to polyvinylidene fluoride membranes. The membranes were blocked with $3 \%$ BSA in Tris-buffered saline containing 0.1\% Tween 20 (TBST) buffer for $4 \mathrm{~h}$ at room temperature. Subsequently, the membranes were incubated overnight at $4^{\circ} \mathrm{C}$ with primary antibodies anti-phosphorylated (P)-AMPK (1:500), anti-AMPK (1:500) anti-SREBP-1 (1:300), anti-FAS (1:800), anti-SREBP-2 (1:500), anti-HMGCR (1:800) and anti- $\beta$-actin (1:800 dilution). Following incubation with the primary antibodies, the membranes were subjected to three 15-min washes with TBST and then incubated with horseradish peroxidase-conjugated secondary anti-mouse antibody and anti-rabbit antibody (1:3,000 dilution) at room temperature for $2 \mathrm{~h}$. Protein bands were visualized using the Enhanced Chemiluminescence Plus substrate (Azure Biosystems, Inc.). All Western blot assays were performed at least three times. The resulting images were analyzed using the ImageJ software (version 1.51; National Institutes of Health) with $\beta$-actin as the loading control.

Statistical analysis. Data were processed using SPSS v18.0 statistical software (SPSS Inc.), and the measurement data 
A

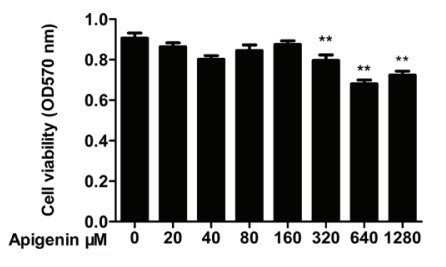

D
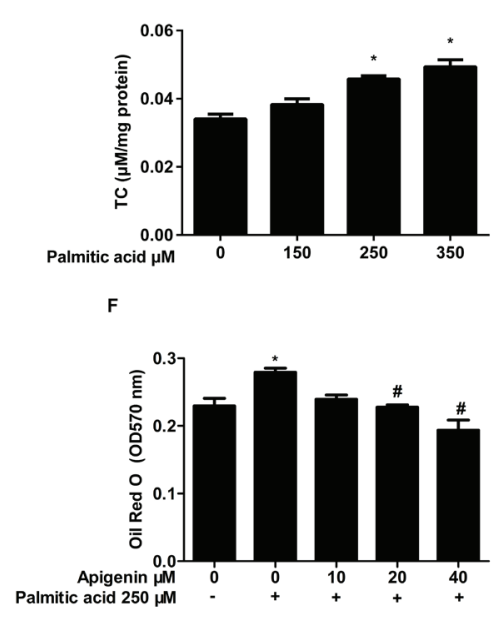

B

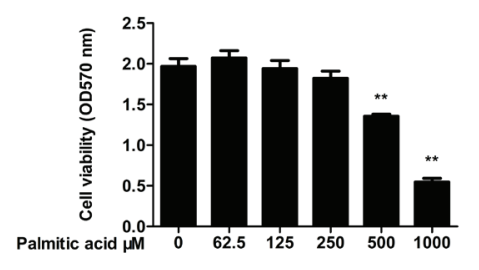

C

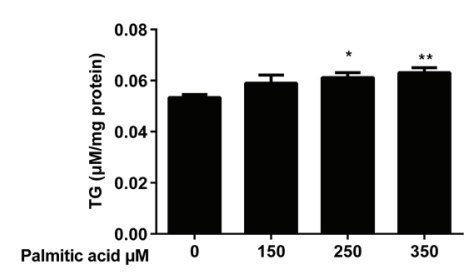

$E$
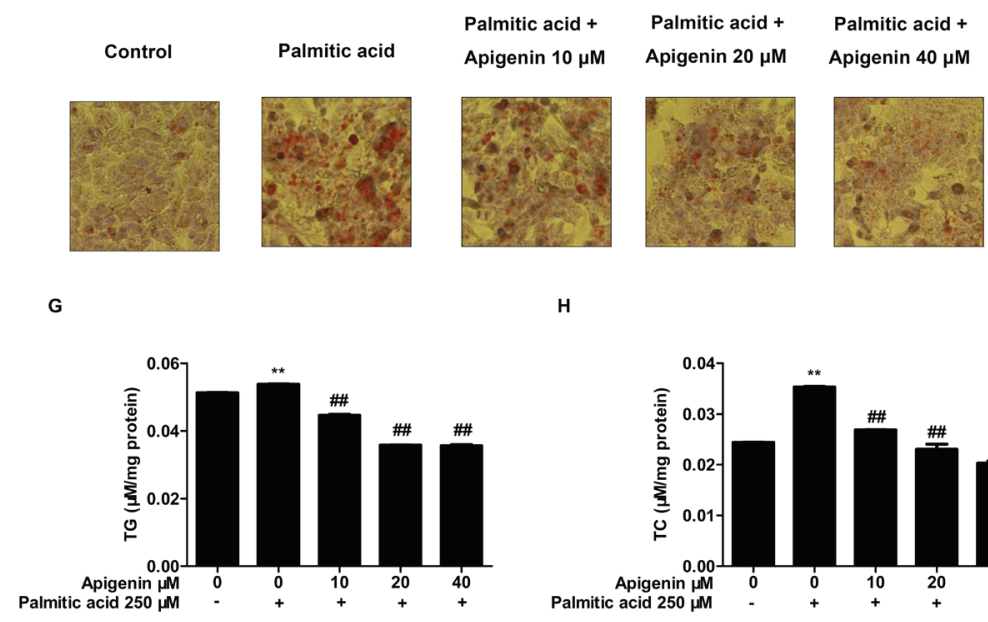

H

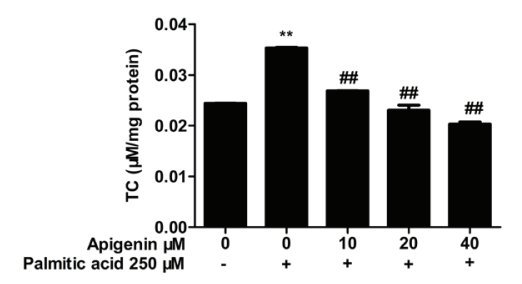

Figure 1. Effects of apigenin and palmitic acid on the viability of HepG2 cells, and analysis of apigenin-mediated inhibition of lipid accumulation in HepG2 cells. (A) Cell viability following treatment with various concentrations of apigenin $(0,20,40,80,160,320,640$ and $1,280 \mu \mathrm{M})$ and (B) palmitic acid $(0,62.5$, $125,250,500$ and $1,000 \mu \mathrm{M})(\mathrm{n}=6)$. (C) TG and (D) TC contents following treatment with various concentrations of palmitic acid (150, 250 and $350 \mu \mathrm{m})$. (E) Photomicrographs of Oil Red O-stained, intracellular lipids (red staining) were obtained by light microscopy (magnification, $\mathrm{x} 400$ ) and (F) quantification of Oil Red O absorbance. (G) TG and (H) TC contents following treatment with palmitic acid (0 or $250 \mathrm{~mm})$ various concentrations of apigenin $(0,10,20$ and $40 \mathrm{~mm} ; \mathrm{n}=3)$. ${ }^{*} \mathrm{P}<0.05$ and ${ }^{* *} \mathrm{P}<0.01$ vs. control; ${ }^{*} \mathrm{P}<0.05$ and ${ }^{\# \#} \mathrm{P}<0.01$ vs. treatment with palmitic acid alone. TG, triglyceride; TC, total cholesterol.

were expressed as the mean \pm standard deviation. Statistical analysis was performed by one-way analysis of variance with Tukey's test and Dunnett's test used for post hoc analysis. Histograms were plotted by Prism software v5.0 (GraphPad Software, Inc.). $\mathrm{P}<0.05$ was considered to indicate a statistically significant difference.

\section{Results}

Effects of apigenin and palmitic acid on cell viability. To analyze the effect of apigenin and palmitic acid on the viability of HepG2 cells, the cells were exposed to different concentrations of apigenin or palmitic acid for $24 \mathrm{~h}$. Results demonstrated that apigenin significantly decreased the viability of HepG2 cells at concentrations from 320 to $1,280 \mu \mathrm{M}(\mathrm{P}<0.01$; Fig. 1A), and palmitic acid significantly decreased the viability of HepG2 cells at concentrations from 500 to $1,000 \mu \mathrm{M}(\mathrm{P}<0.01$; Fig. 1B).

Effects of apigenin on lipid accumulation in palmitic acid-treated Hep 2 cells. The cells were exposed to different concentrations of palmitic acid $(150,250$ and $350 \mu \mathrm{M})$ for $24 \mathrm{~h}$ and cellular TC and TG contents were subsequently measured. The results demonstrated that the intracellular TC and TG levels were significantly elevated when cells were treated with 250 and $350 \mu \mathrm{M}$ palmitic acid compared with control cells $(\mathrm{P}<0.05$; Fig. 1C and D). The accumulation of lipid droplets in cells was measured by Oil red $\mathrm{O}$ staining. The results indicated that the lipid content in HepG2 cells treated with $250 \mu \mathrm{M}$ palmitic acid was increased significantly compared with the control cells. In addition, 20 and $40 \mu \mathrm{M}$ apigenin treatment significantly decreased the level of the lipid content compared with treatment with palmitic acid alone $(\mathrm{P}<0.05$; Fig. 1E and F).

To verify the Oil red $\mathrm{O}$ staining results, the cellular TC and TG contents were measured. Results demonstrated that the intracellular TC and TG levels were significantly elevated when cells were treated with $250 \mu \mathrm{M}$ palmitic acid compared with the control cells $(\mathrm{P}<0.01$; Fig. $1 \mathrm{G}$ and $\mathrm{H})$. However, treatment with $250 \mu \mathrm{M}$ palmitic acid and different concentrations of apigenin $(10,20$ and $40 \mu \mathrm{M})$ significantly suppressed intracellular TC and TG levels in a concentration-dependent manner $(\mathrm{P}<0.01$; Fig. $1 \mathrm{G}$ and $\mathrm{H})$.

Effects of apigenin on the AMPK and P-AMPK proteins in palmitic acid-induced HepG2 cells. The activity of AMPK is closely associated with phosphorylation of the Thr172 site in the $\alpha$ subunit (P-AMPK $\alpha 1$ ). Therefore, the activity of AMPK can be evaluated by measuring the levels of P-AMPK $\alpha$ protein. Results demonstrated that the P-AMPK/AMPK ratio in the cells treated with $250 \mu \mathrm{M}$ palmitic acid was significantly lower than in the normal group $(\mathrm{P}<0.01$; Fig. 2A). However, the treatment of HepG2 cells with $250 \mu \mathrm{M}$ palmitic acid and different concentrations of apigenin (10, 20 and $40 \mu \mathrm{M})$ 
A

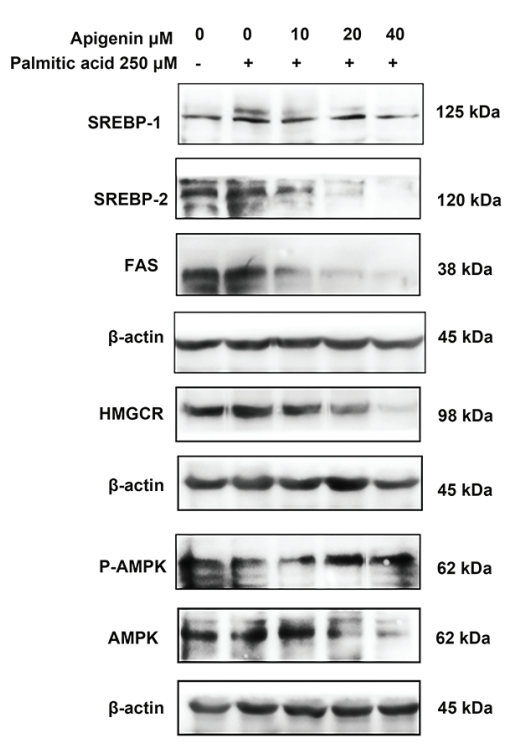

B

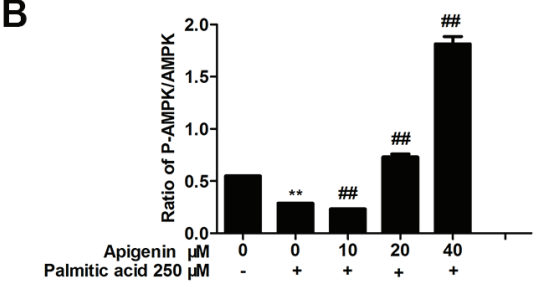

C

D
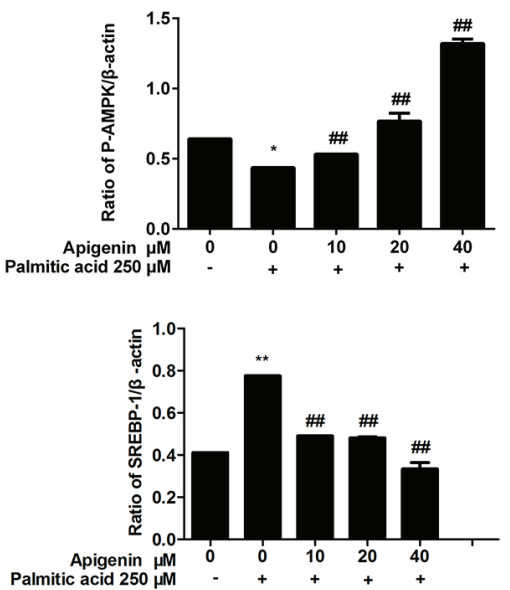

E

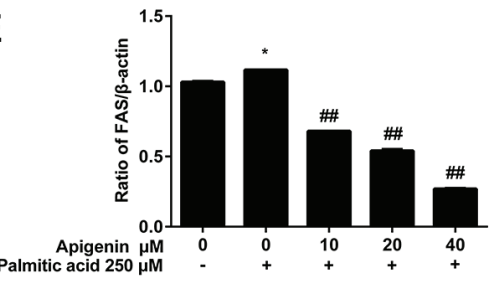

F

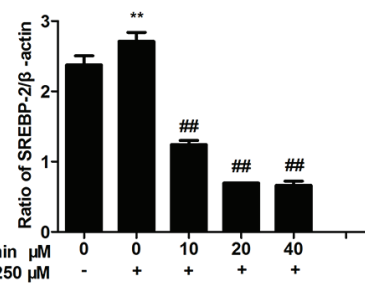

G

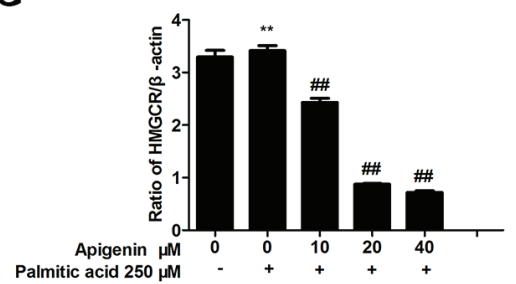

Figure 2. Effect of apigenin on the expression of AMPK, P-AMPK, SREBP-1, FAS, SREBP-2 and HMGCR proteins in HepG2 cells. (A) Representative western blots and (B) quantification of P-AMPK/AMPK, (C) P-AMPK/ $\beta$-actin, (D) SREBP-1, (E) FAS, (F) SREBP-2 and (G) HMGCR protein expression measured by western blot analysis $(n=3) .{ }^{*} \mathrm{P}<0.05$ and ${ }^{* *} \mathrm{P}<0.01$ vs. control; ${ }^{\# \#} \mathrm{P}<0.01$ vs. treatment with palmitic acid alone. AMPK, AMP-activated protein kinase; P-AMPK, phosphorylated AMPK; SREBP, sterol regulatory element-binding protein; FAS, fatty acid synthase; HMGCR, 3-hydroxy-3-methylglutaryl CoA reductase.

significantly increased AMPK phosphorylation $(\mathrm{P}<0.01$ Fig. 2A). The P-AMPK/ $\beta$-actin ratio in the cells treated with $250 \mu \mathrm{M}$ palmitic acid and different concentrations of apigenin $(10,20$ and $40 \mu \mathrm{M})$ produced the same results as aforementioned ( $\mathrm{P}<0.01$; Fig. 2B).

Effects of apigenin on the AMPK/SREBP signaling pathway in palmitic acid-treated HepG2 cells. To investigate the mechanism underlying the apigenin-mediated inhibition of lipid production and regulation of the AMPK signaling pathway in HepG2 cells, the expression levels of proteins associated with fatty acid synthesis and cholesterol synthesis were determined.

Results demonstrated that compared with the control cells, the levels of SREBP-1 ( $\mathrm{P}<0.01$; Fig. 2B) and FAS $(\mathrm{P}<0.05$; Fig. 2C) increased significantly following treatment with $250 \mu \mathrm{M}$ palmitic acid, whereas the levels of SREBP-1 and FAS were decreased by apigenin in a concentration-dependent manner $(\mathrm{P}<0.01$; Fig. $2 \mathrm{~B}$ and $\mathrm{C})$. SREBP-2 and HMGCR protein expression increased significantly upon treatment with $250 \mu \mathrm{M}$ palmitic acid compared with the control $(\mathrm{P}<0.01$; Fig. 2D and E). However, HepG2 cells treated with $250 \mu \mathrm{M}$ palmitic acid and different concentrations of apigenin (10, 20 and $40 \mu \mathrm{M}$ ) inhibited the upregulation of SREBP-2 and HMGCR protein expression ( $\mathrm{P}<0.01$; Fig. $2 \mathrm{D}$ and $\mathrm{E})$.

Effects of compound $C$ on lipid accumulation and AMPK expression in palmitic acid-treated HepG2 cells. To further validate the previous findings, the intracellular TC and TG contents were measured following pretreatment with $10 \mu \mathrm{M}$ compound $\mathrm{C}$ for $1 \mathrm{~h}$. The compound $\mathrm{C}$ treatment significantly reversed the apigenin-induced reductions in lipid accumulation and led to increases in TC and TG contents $(\mathrm{P}<0.01$; Fig. 3A and B).

In addition, the protein expression of AMPK and P-AMPK were examined. The increase in P-AMPK/AMPK ratio induced by apigenin was reversed by pretreatment with compound $\mathrm{C}$ in HepG2 cells ( $\mathrm{P}<0.01$; Fig. $3 \mathrm{C}$ and $\mathrm{D})$. These results indicated that apigenin activated the AMPK signaling pathway in HepG2 cells.

The present findings indicated that apigenin effectively inhibited the effects of palmitic acid-induced lipid accumulation via the AMPK/SREBP signaling pathway in HepG2 cells (Fig. 4).

\section{Discussion}

Numerous flavonoids display anti-obesity and hypolipidemic effects in animals and humans. It has been demonstrated that apigenin exerts anticancer, anti-inflammatory and hypotensive effects (10-12). Previous studies have determined that apigenin reduces the accumulation of intracellular lipids in 3T3-L1 cells (18), and also attenuates atherogenesis via upregulating ATP binding cassette subfamily A member 1-mediated cholesterol efflux and by inhibiting inflammation (19). Apigenin suppresses adipogenesis through the downregulation of peroxisome proliferator activated receptor- $\gamma$ function by activating AMPK in 3T3-L1 cells (4). Nonetheless, the regulatory mechanism underlying the apigenin-mediated suppression of lipid accumulation has yet to be fully identified. 
A

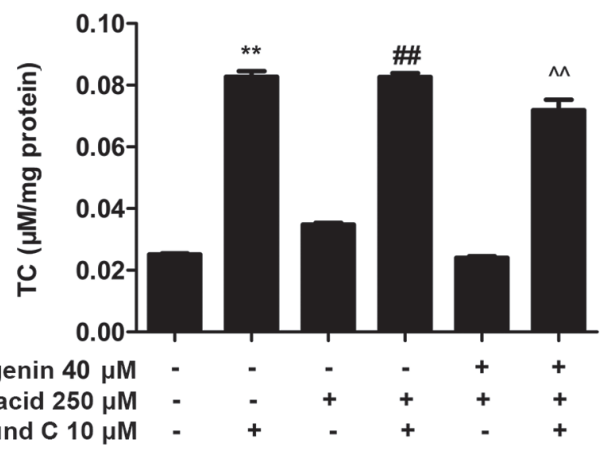

C

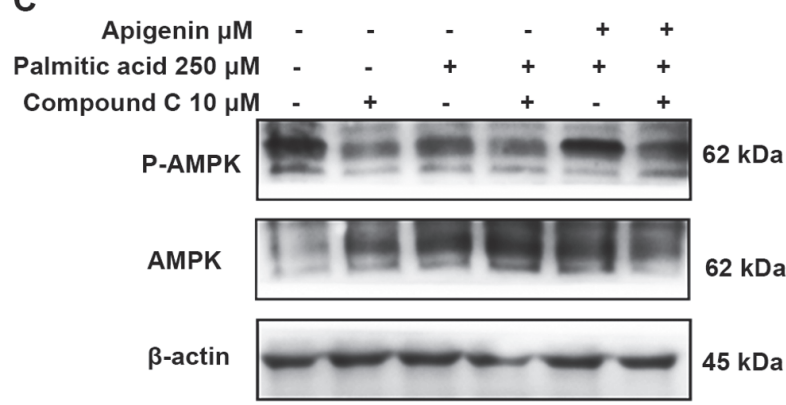

B

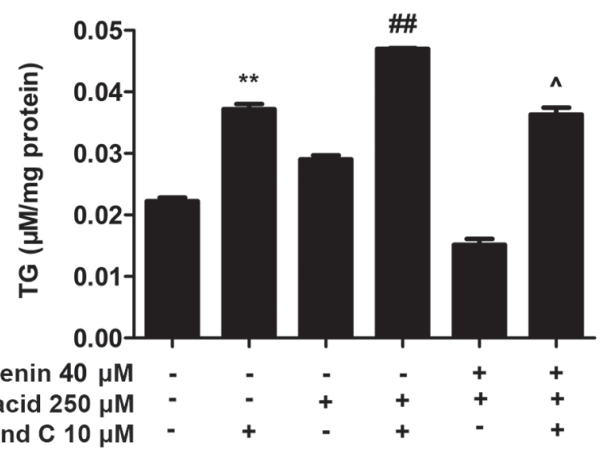

D

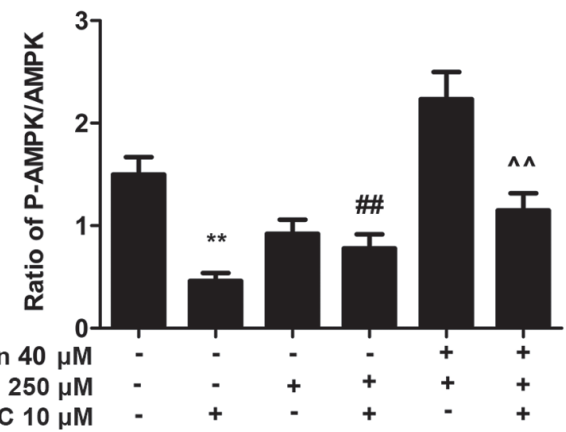

Figure 3. Effect of an AMPK inhibitor compound C on AMPK and P-AMPK protein levels, and the apigenin-mediated inhibition of lipid accumulation in HepG2 cells. (A) TC and (B) TG contents following treatment with palmitic acid (0 or $250 \mathrm{~mm})$, apigenin $(0$ or $40 \mathrm{~mm})$ and compound C (0 or $10 \mathrm{~mm})$. (C) Representative western blots and (D) quantification of P-AMPK protein levels determined by western blot analysis $(\mathrm{n}=3)$. ${ }^{* *} \mathrm{P}<0.01 \mathrm{vs}$. control; ${ }^{\# \#} \mathrm{P}<0.01 \mathrm{vs}$. treatment with palmitic acid alone; ${ }^{\wedge} \mathrm{P}<0.05$ and ${ }^{\wedge} \mathrm{P}<0.01$ vs. treatment with apigenin and palmitic acid. AMPK, AMP-activated protein kinase; $p$, phosphorylated; TG, triglyceride; TC, total cholesterol.

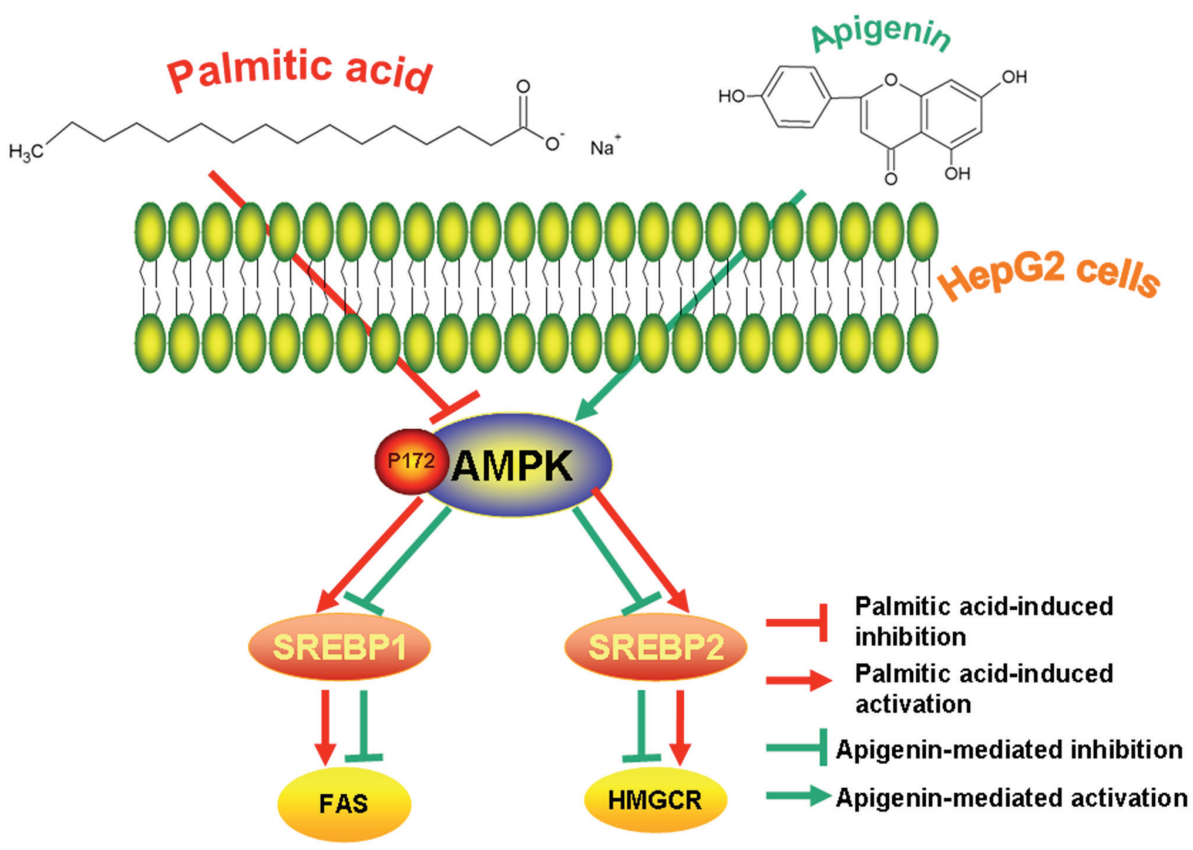

Figure 4. Hypothetical mechanism underlying the inhibition of palmitic acid-induced lipid accumulation by apigenin in HepG2 cells. AMPK, AMP-activated protein kinase; SREBP, sterol regulatory element-binding protein; FAS, fatty acid synthase; HMGCR, 3-hydroxy-3-methylglutaryl CoA reductase.

The present study identified that apigenin served an essential role in the reduction of excessive lipid accumulation induced by palmitic acid in HepG2 cells. Results demonstrated that apigenin was not toxic to HepG2 cells at concentrations of $<320 \mu \mathrm{M}$, whilst palmitic acid exhibited no apparent toxicity to HepG2 cells at concentrations $<250 \mu \mathrm{M}$. Of note, apigenin has been determined to be cytotoxic to 3T3-L1 cells at concentrations as low as $10 \mu \mathrm{M}(4)$, whilst palmitic acid is cytotoxic to HepG2 cells at concentrations as low as $200 \mu \mathrm{M}$, which is in line with the present study (20). The discrepancies might be due to differences between cell lines or assay systems with further investigation required to elucidate a clear explanation. 
A previous study demonstrated that apigenin is a poorly soluble bioactive compound/nutraceutical with a poor bioavailability upon oral administration (21). The oral bioavailability of apigenin is relatively poor due to its low lipid $(0.001-1.63 \mathrm{mg} / \mathrm{ml}$ in nonpolar solvents) and water $(2.16 \mu \mathrm{g} / \mathrm{ml}$ in water) solubility, which has severely limited its development for clinical applications $(22,23)$. However, there are a number of methods in which the bioavailability of apigenin can be improved, including utilizing spray-drying techniques and apigenin-loaded polymeric micelles $(24,25)$. Therefore, future research into apigenin should not only take into consideration the lower reported toxic dose but also try to improve the oral bioavailability of apigenin.

To further elucidate the molecular mechanism underlying the apigenin-mediated suppression of adipogenesis, the present study investigated the activation of AMPK, which is an important kinase that regulates energy homeostasis. This key protein is involved in various cell signal transduction pathways, senses the intracellular energy state and has an important role in the regulation of glucose and lipid metabolism (26,27). AMPK activation reportedly leads to changes in obesity and has been linked to obesity prevention $(28,29)$. Therefore, the pharmacological activity of AMPK is expected to have an important role in the study of apigenin-induced liver lipid accumulation reduction. This study identified that cotreatment of apigenin (10, 20 and $40 \mu \mathrm{M}$ ) and $250 \mu \mathrm{M}$ palmitic acid led to a dose-dependent upregulation of the P-AMPK/AMPK ratio compared with the group treated with $250 \mu \mathrm{M}$ palmitic acid alone. This indicated that apigenin activated AMPK phosphorylation in the palmitic acid-induced HepG2 cells. Thus, to further validate the experimental results, cells were treated with the AMPK inhibitor compound $\mathrm{C}$. The increase in the P-AMPK/AMPK ratio induced by apigenin in HepG 2 cells was inhibited by pretreatment with compound $\mathrm{C}$ in HepG2 cells. Therefore, apigenin inhibited excessive lipid accumulation induced by palmitic acid by activating AMPK phosphorylation.

Changes in proteins associated with the AMPK/SREBP pathway were investigated to fully elucidate the hypolipidemic mechanism of apigenin. Apigenin treatment activates AMPK thereby further inhibiting the synthesis of fatty acids and cholesterol (8). It has been demonstrated that AMPK inhibits SREBP-1 and SREBP-2 and thereby reduces the synthesis of cholesterol, fatty acids, and triglycerides in the liver (30). Lipid production in the liver is mainly regulated by SREBP-1, which is a crucial regulatory factor in fatty acid synthesis. SREBP-1 regulates the expression of other genes involved in TG synthesis, such as FAS (31). SREBP-1c inhibition reduces the expression of FAS and alleviates fat-containing liver lesions in mice (32). Similarly, SREBP-2 is mainly involved in the regulation of cholesterol synthesis, and HMGCR is a target gene of SREBP-2 (33). Decreased SREBP-2 activity can lead to reduced HMGCR expression. In addition, studies have identified that the presence of free cholesterol significantly increases the levels of SREBP-2 and its target gene HMGCR in the livers of hyperlipidemic mice $(34,35)$. These studies indicate that the changes in the levels of proteins in the AMPK/SREBP pathway are correlated with the TC and TG levels. In the present study, compared with palmitic acid treatment alone, cotreatment with different concentrations of apigenin (10, 20 and $40 \mu \mathrm{M})$ and palmitic acid significantly suppressed TC and TG levels, as well as SREBP-1, FAS, SREBP-2, and HMGCR protein expression. Taken together, these results demonstrated that apigenin decreased lipid levels and alleviated the palmitic acid-induced accumulation of lipids in HepG2 cells by activating the AMPK/SREBP signaling pathway.

In summary, apigenin alleviated the palmitic acid-induced increases in lipid accumulation in HepG2 cells thereby reducing the lipid content. The underlying mechanism might be associated with the activation of the intracellular AMPK/SREBP signaling pathway. These findings provide a novel approach for the development of natural hypolipidemic drugs to prevent lipid accumulation.

\section{Acknowledgements}

Not applicable.

\section{Funding}

This study was funded by the Graduate Innovation Fund of Jilin University, China (grant no. 419020201348).

\section{Availability of data and materials}

All data generated or analyzed during this study are included in this published article.

\section{Authors' contributions}

SG, JL, ZM and BC conceived the study and participated in its design. ZM and BC performed the experiments. ZM, BC, ML and ST participated in the acquisition and interpretation of the data. SG, JL, ZM and BC participated in manuscript drafting. All authors read and approved the final manuscript.

\section{Ethics approval and consent to participate}

Not applicable.

\section{Patient consent for publication}

Not applicable.

\section{Competing interests}

The authors declare that they have no competing interests.

\section{References}

1. Williams JA, Manley S and Ding WX: New advances in molecular mechanisms and emerging therapeutic targets in alcoholic liver diseases. World J Gastroenterol 20: 12908-12933, 2014.

2. Wan Y, Liu LY, Hong ZF and Peng J: Ethanol extract of Cirsium japonicum attenuates hepatic lipid accumulation via AMPK activation in human HepG2 cells. Exp Ther Med 8: 79-84, 2014.

3. Zhang M, Yuan Y, Wang Q, Li X, Men J and Lin M: The Chinese medicine Chai $\mathrm{Hu} \mathrm{Li}$ Zhong Tang protects against non-alcoholic fatty liver disease by activating AMPK $\alpha$. Biosci Rep 38: BSR20180644, 2018.

4. Ono $\mathrm{M}$ and Fujimori K: Antiadipogenic effect of dietary apigenin through activation of AMPK in 3T3-L1 cells. J Agric Food Chem 59: 13346-13352, 2011.

5. Xiao X and Song BL: SREBP: A novel therapeutic target. Acta Biochim Biophys Sin 45: 2-10, 2013. 
6. Shimano H: Sterol regulatory element binding proteins (SREBPs): Transcriptional regulators of lipid synthetic genes. Prog Lipid Res 40: 439-452, 2001.

7. Wong TY, Lin SM and Leung LK: The flavone luteolin suppresses SREBP-2 expression and post-translational activation in hepatic cells. PLoS One 10: e0135637, 2015.

8. Mottillo EP, Desjardins EM, Crane JD, Smith BK, Green AE, Ducommun S, Henriksen TI, Rebalka IA, Razi A, Sakamoto K, et al: Lack of adipocyte AMPK exacerbates insulin resistance and hepatic steatosis through brown and beige adipose tissue function. Cell Metab 24: 118-129, 2016.

9. Lu J, Cheng B, Meng Z, Sun M, Fang B, Li T, Sun M, Liu M and Guan S: Alliin attenuates 1,3-dichloro-2-propanol-induced lipogenesis in HepG2 cells through activation of the AMP-activated protein kinase-dependent pathway. Life Sci 195: 19-24, 2018.

10. Lordan S, O'Neill C and O'Brien NM: Effects of apigenin, lycopene and astaxanthin on 7 beta-hydroxycholesterol-induced apoptosis and Akt phosphorylation in U937 cells. Br J Nutr 100: 287-296, 2008.

11. Tong $X$ and Pelling J: Targeting the PI3K/Akt/mTOR axis by apigenin for cancer prevention. Anticancer Agents Med Chem 13: 971-978, 2013

12. Zhu ZY, Gao T, Huang Y, Xue J and Xie ML: Apigenin ameliorates hypertension-induced cardiac hypertrophy and down-regulates cardiac hypoxia inducible factor-l $\alpha$ in rats. Food Funct 7: 1992-1998, 2016

13. Ren B, Qin W, Wu F, Wang S, Pan C, Wang L, Zeng B, Ma S and Liang J: Apigenin and naringenin regulate glucose and lipid metabolism, and ameliorate vascular dysfunction in type 2 diabetic rats. Eur J Pharmacol 773: 13-23, 2016.

14. Escande C, Nin V,Price NL, Capellini V, Gomes AP, Barbosa MT, O'Neil L, White TA, Sinclair DA and Chini EN: Flavonoid apigenin is an inhibitor of the NAD+ ase CD38: Implications for cellular NAD+ metabolism, protein acetylation, and treatment of metabolic syndrome. Diabetes 62: 1084-1093, 2013

15. Poitout V and Robertson RP: Glucolipotoxicity: Fuel excess and beta-cell dysfunction. Endocr Rev 29: 351-366, 2008.

16. Mayer CM and Belsham DD: Palmitate acid attenuates insulin signaling and induces endoplasmic reticulum stress and apoptosis in hypothalamic neurons: The rescue of resistance and apoptosis through adenosine 5 ' monophosphate-activated protein kinase activation. Endocrinology 151: 576-585, 2010.

17. Park JY, Kim Y, Im JA and Lee H: Oligonol suppresses lipid accumulation and improves insulin resistance in a palmitate-induced in HepG2 hepatocytes as a cellular steatosis model BMC Complement Altern Med 15: 185, 2015

18. Kim J, Lee I, Seo J, Jung M, Kim Y, Yim N and Bae K: Vitexin, orientin and other flavonoids from Spirodela polyrhiza inhibit adipogenesis in 3T3-L1 cells. Phytother Res 24: 1543-1548, 2010.

19. Ren K, Jiang T, Zhou HF, Liang Y and Zhao GJ: Apigenin retards atherogenesis by promoting ABCA1-mediated cholesterol efflux and suppressing inflammation. Cell Physiol Biochem 47: 2170-2184, 2018

20. Vock C, Gleissner M, Klapper M and Döring F: Identification of palmitate-regulated genes in HepG2 cells by applying microarray analysis. Biochim Biophys Acta 1770: 1283-1288, 2007.

21. Zechner R, Zimmermann R, Eichmann TO, Kohlwein SD, Haemmerle G, Lass A and Madeo F: FAT SIGNALS-lipases and lipolysis in lipid metabolism and signaling. Cell Metab 15: 279-291, 2012
22. Block MJ: American chemical society. Chemcycolpedia 2000 106-107, 2001.

23. Zhang J, Liu D, Huang Y, Gao Y and Qian S: Biopharmaceutics classification and intestinal absorption study of apigenin. Int J Pharm 436: 311-317, 2012.

24. Altamimi MA, Elzayat EM, Alshehri SM, Mohsin K, Ibrahim MA, Al Meanazel OT, Shakeel F, Alanazi FK and Alsarra IA: Utilizing spray drying technique to improve oral bioavailability of apigenin. Adv Powder Technol 29: 1676-1684 2018.

25. Zhai YJ, Guo SS, Liu CH, Yang CF, Dou JF, Li LB and Zhai GX: Preparation and in vitro evaluation of apigenin-loaded polymeric micelles. Colloids Surfaces A: Physicochemical Engineering Aspects 429: 24-30, 2013

26. Hardie DG: Minireview: The AMP-activated protein kinase cascade: The key sensor of cellular energy status. Endocrinology 144: 5179-5183, 2003.

27. Carling D: The AMP-activated protein kinase cascade-a unifying system for energy control. Trends Biochem Sci 29: 18-24, 2004

28. Kang MC, Ding Y, Kim EA, Choi YK, de Araujo T, Heo SJ and Lee SH: Indole derivatives isolated from Brown alga Sargassum thunbergii inhibit Adipogenesis through AMPK activation in 3T3-L1 preadipocytes. Mar Drugs 15: E119, 2017.

29. Long YC and Zierath JR: AMP-activated protein kinase signaling in metabolic regulation. J Clin Invest 116: 1776-1783, 2006.

30. Ruderman N and Prentki M: AMP kinase and malonyl-CoA: Targets for therapy of the metabolic syndrome. Nat Rev Drug Discov 3: 340-351, 2004.

31. Sun X, Duan X, Wang C, Liu Z, Sun P, Huo X, Ma X, Sun H, Liu K and Meng Q: Protective effects of glycyrrhizic acid against non-alcoholic fatty liver disease in mice. Eur J Pharmacol 5: 75-82, 2017.

32. Frederico MJ, Vitto MF, Cesconetto PA, Engelmann J, De Souza DR, Luz G, Pinho RA, Ropelle ER, Cintra DE and De Souza CT: Short-term inbibition of SREBP-1c expression reversed dietinduced nonalcoholic fatty liver disease in mice. Scand J Gastroenterol 46: 1381-1388, 2011.

33. Sakakura Y, Shimano H, Sone H, Takahashi A, Inoue $\mathrm{N}$, Toyoshima H, Suzuki S and Yamada N: Sterol regulatory element-binding proteins induce an entire pathway of cholesterol synthesis. Biochem Biophys Res Commun 286: 176-183, 2001.

34. Caballero F, Fernandez A, De-Lacy AM, Fernandez-Checa JC, Caballeria J and Garcia-Ruiz C: Enhanced free cholesterol, SREBP-2 and StAR expression in human NASH. J Hepatol 50: 789-796, 2009

35. Sun JH, Liu X, Cong LX, Li H, Zhang CY, Chen JG and Wang CM: Metabolomics study of therapeutic mechanism of Schisandra Chinensislignans in diet-induced hyperlipidemia mice. Lipids Health Dis 16: 145, 2017.

This work is licensed under a Creative Commons Attribution-NonCommercial-NoDerivatives 4.0 International (CC BY-NC-ND 4.0) License. 